\title{
Aggregation properties of triamcinolone acetonide injection in human serum: considerations when performing epidural steroid injections
}

This article was published in the following Dove Medical Press journal: Journal of Pain Research

\author{
Sayed E Wahezi' \\ Salah Eldin Mohamed ${ }^{2}$ \\ Andrew Lederman' \\ Amanda P Beck ${ }^{3}$ \\ 'Montefiore Medical Center, Albert \\ Einstein College of Medicine, Bronx, \\ NY, USA; '²urke Rehabilitation \\ Hospital, Albert Einstein College \\ of Medicine, White Plains, NY, USA \\ ${ }^{3}$ Department of Pathology, Albert \\ Einstein College of Medicine, Bronx, \\ NY, USA
}

\begin{abstract}
Background: Morbidity has been reported as a sequelae of crystalline steroid epidural steroid injections (ESIs), and particulate steroid size, aggregation, and embolization in brain and spinal cord may be the mechanism related to these neurologic effects.

Objective: The objective of the study was to examine the aggregation properties of triamcinolone acetonide in commonly used local anesthetics with and without human serum.

Setting: This study was conducted in an academic tertiary care center.

Hypothesis: Triamcinolone acetonide shows different aggregation characteristics in serum compared to a non-physiologic solution.

Design: Triamcinolone acetonide was mixed with lidocaine 1\% (first group) and bupivacaine $0.5 \%$ (second group) in a 1:1 ratio and then mixed with either distilled water (control group) or serum ex vivo. A pathologist blinded to our hypothesis inspected all solutions under light microscopy with $100 \times$ and $400 \times$ magnifications. Total number of particulate steroid aggregates and the number of particles forming each aggregate (recorded as single, ${ }^{1}$ double,${ }^{2}$ triple, ${ }^{3}$ quadruple, ${ }^{4}$ or large $[>4\}$ crystals) were counted. Particle size and aggregate size were measured (in $\mu \mathrm{m}$ ). The ratios of quadruple to total aggregates, large to total, and quadruple with large to total aggregates were calculated. Steroid-serum solutions and steroid-sterile water were then compared.
\end{abstract}

Results: Triamcinolone aggregates showed an increased crystal and aggregate size when compared with other steroids. Within the triamcinolone subgroup, the mixture of lidocaine $1 \%$ and serum resulted in the largest crystal aggregates.

Limitations: Whole blood analysis may have provided a more physiologically accurate model but was not chosen due to poor microscopic analysis. Serum donor variability may also have affected particle characteristics.

Conclusion: Fewer large triamcinolone aggregates were noted in the presence of serum when compared to the non-serum control groups. However, when compared to previously studied particulate steroids, it had the largest aggregates when added to serum.

Keywords: triamcinolone, betamethasone, aggregation, lidocaine 1\%, methylprednisolone

\section{Introduction}

There are several options of corticosteroids for epidural steroid injection (ESI). Triamcinolone has recently been criticized for a possible link between spinal cord and brain infarcts when injected into the epidural space. ${ }^{1,2}$ Embolization of particulate cor-
Correspondence: Sayed E Wahezi Montefiore Medical Center, Albert Einstein College of Medicine, III Eas 210th Street, Bronx, NY 10467-240I, USA

$\mathrm{Tel}+\mathrm{I} 7189207246$

Fax +I 7189202287

Email swahezi@montefiore.org 
ticosteroids in transforaminal injection leading to spinal cord ischemia is the suggested mechanism, and some consider vasospasm of the anterior spinal artery (ASA) or radiculomedullary artery an alternate explanation. ${ }^{3-6}$ Embolization is the favored theory in events where particulate steroids have been used. This may be because a number of investigators have demonstrated aggregation of triamcinolone in commonly prepared ESI solutions. ${ }^{7-9}$ Based on these basic science and clinical literature reports, the United States Food and Drug Administration released a warning against the use of particulate steroids when performing spinal injections in April 2014. ${ }^{10}$

The rates of ESI performance in the United States is very high, but devastating complications are rare. ${ }^{1}$ In a previous paper, our team attempted to explain this discrepancy when we evaluated the behavior of clumping patterns of particulate steroids in human serum. We hypothesized that particulate steroid behavior is different in physiologic solutions compared to non-physiologic solutions. We demonstrated that betamethasone becomes significantly less particulate when in serum, but methylprednisolone does not. We did not examine triamcinolone because we assumed that the reported disproportionate ratio of devastating outcomes with triamcinolone compared to other particulate steroids is very high. ${ }^{11}$ Hundreds of thousands of ESIs are performed in the United States every year and less than a dozen associated neurologic events are reported. ${ }^{12}$ However, many pain physicians believe that even this number is too high for, what is thought to be, an innocuous procedure. The heightened awareness that triamcinolone may be a potentially dangerous choice for spinal injections has prompted us to evaluate the aggregation behavior of triamcinolone in serum. Clumping profile of triamcinolone did not reduce in serum, validating the potentially devastating effects this medication can have if injected into the spinal cord or cranial vasculature.

\section{Methods}

About $0.5 \mathrm{~mL}$ of base triamcinolone (Kenalog $40 \mathrm{mg} / \mathrm{mL}$ ) solutions were prepped with equal volumes of distilled water or serum using micropipettes. Each water and serum base was then mixed separately. To each distilled water and serum base solution, $1 \%$ lidocaine or $0.5 \%$ bupivacaine was added in a 1:1 ratio using micropipettes for dilution accuracy. Each aliquot was prepared separately and placed onto a glass histology slide. All solutions were prepared on site and examined immediately (within 30 seconds of mixing) via light microscopy under $100 \times$ and $400 \times$ magnification by a blinded board-certified histologist. All groups were kept at $37^{\circ} \mathrm{C}$ during preparation and pipetting, but exposed to room temperature of $23.3^{\circ} \mathrm{C}$ for no more than $2-3$ minutes. Rapid transfer and evaluation were performed to lessen the impact of room temperature on physiologic temperature of aliquots.

The serum was drawn from two healthy human subjects (no history of disease and not on any medications) into a standard vacuum sealed blood draw tube without anticoagulation additive. They were then centrifuged at 10,000 rpm for 15 minutes. Subsequently, the serum supernatant was collected and maintained at $37^{\circ} \mathrm{C}$ in a water bath to preserve physiologic integrity of the solution.

Photomicrographs of five randomly selected microscopic fields at $100 \times$ and $400 \times$ magnification containing particles were taken of each preparation and aggregate size was measured. The total number of particulate steroid aggregates and the number of particles forming each aggregate (recorded as either single [1], double [2], triple [3], quadruple [4], or large $[>4\}$ crystals) were counted. The particle and aggregate sizes were then measured (in $\mu \mathrm{m}$ ) in each field. The ratios of quadruple to total aggregates (RQT), large to total (RLT), and quadruple with large to total aggregates (RQLT) were calculated. The steroid-serum solutions were then compared to the steroid-sterile water solution. Lastly, comparison and statistical significance were evaluated using a two-sided Mann-Whitney $U$-test resulting in $\mathrm{Z}$-scores and associated $P$-values.

The human subjects from whom the serum was drawn provided written informed consent in compliance with the Declaration of Helsinki.

\section{Results}

The particle and aggregate sizes were measured (in $\mu \mathrm{m}$ ) in each microscopic field. RQT, RLT, and RQLT were calculated (Table 1). The steroid-serum solutions were then compared to the steroid-sterile water solution.

Dilution of triamcinolone with $1 \%$ lidocaine in serum solution showed a statically significant increase in the RLT (ie, $>4$ particle aggregates) compared to other commonly used particulate steroids for ESIs (Table 1). While statistical significance was not achieved with the RQLT, the $P$-value very closely approached significance $(P=0.057)$. On the other hand, there was no statistical significance in the RQT and RQLT when triamcinolone was diluted in water or $0.5 \%$ bupivacaine $(P>0.05)$. The size of the largest aggregate in each solution was also noted. The largest was $352 \mu \mathrm{m}$ and the smallest was $70 \mu \mathrm{m}$, from water only and $1 \%$ lidocaine preparation, respectively (Figure 1).

Our results demonstrate that while serum without local anesthetic ex vivo will decrease the size of the triamcinolone 
Table I The RQT, RLT, and RQLT between different injectate in serum vs non-serum

\begin{tabular}{|l|l|l|l|l|l|l|}
\hline Prep & RQT & P-value & RLT & $P$-value & RQLT & P-value \\
\hline BetaW & -0.6667 & 0.50286 & -2.8 & 0.005 I2 & -2.9333 & 0.00338 \\
\hline Beta I\% & 0.4 & 0.68916 & -1.6 & 0.1096 & -0.8 & 0.42372 \\
\hline Beta0.5\% & $1.1635 I$ & 0.24604 & -3.0006 & 0.0027 & -2.8782 & 0.00398 \\
\hline MPW & -0.3062 & 0.75656 & -0.6736 & 0.50286 & -0.6736 & 0.50286 \\
\hline MPI\% & 0.06124 & 0.95216 & -2.1433 & 0.03236 & -1.286 & 0.19706 \\
\hline MP0.5\% & -0.9186 & 0.35758 & 2.3883 & 0.01684 & 1.6534 & 0.09894 \\
\hline Triam I\% & -1.3472 & 0.17702 & 2.26578 & 0.0232 & 1.89835 & 0.05744 \\
\hline Triam 0.5\% & $0.158 I I$ & 0.87288 & 0.26352 & 0.79486 & 0.47434 & 0.63836 \\
\hline Triam W & 0.9798 & 0.32708 & -1.8371 & 0.6576 & $-I .5922$ & 0.11184 \\
\hline
\end{tabular}

Abbreviations: Beta, betamethasone; Bup, bupivacaine; Lido, lidocaine; MP, methylprednisolone; prep, preparation; RLT, ratio of large to total; RQLT, ratio of quadruple with large to total aggregates; RQT, ratio of quadruple to total aggregates; Triam, triamcinolone; $\mathrm{W}$, water.

particulates, a serum-based solution with triamcinolone and $1 \%$ lidocaine had a significant increase in the RLT $(P<0.05)$. In other words, the combination of triamcinolone with $1 \%$ lidocaine in serum resulted in a high proportion of particle aggregates composed of more than four particles each. This is demonstrated in Figure 2, which illustrates an increase in triamcinolone aggregation in serum/local anesthetic solution, compared to non-physiologic solutions with local anesthetic.

\section{Discussion}

ESIs are commonly used to treat a variety of spine pain conditions. Generally accepted as an innocuous procedure, rare, but devastating, associated complications have been reported. ${ }^{12}$ Despite the infrequency of such cases, much attention has been directed at the safety of these procedures. There are a number of published reports regarding their complications, including death, spinal cord, vertebrobasilar, and brain infarcts. ${ }^{13,14}$ Arterial embolization with particulate steroid is the well-accepted mechanism; therefore, epidurals performed in spinal locations with dense local arterial flow are most vulnerable to these devastating outcomes. Available case series reports support this theory, as the incidence of severe neurological deficits are disproportionally more common with cervical transforaminal epidural spinal injections than with epidurals performed in areas with less prominent arterial structures. ${ }^{15}$ This theory of intra-arterial particulate steroid injections causing embolization has also been supported in the literature. ${ }^{16-21}$ In addition, many investigators believe that insoluble particulates, such as steroids injected during ESIs, increase the risk for poor neurological sequelae. Benzon et al validated this causal risk for ESI and steroid embolus by comparing the histological size of commonly used particulate steroids with and without the solutions they are mixed with prior to injection..$^{22}$ Their investigation clearly demonstrated that particulate steroids could occlude a small- to medium-sized arteriole. It has also been reported that occult intra-arterial injection during ESIs are much more common than the reported neurological sequelae..$^{23} \mathrm{Nahm}$ et al described a $20 \%$ incidence of intravascular injection during the performance of cervical transforaminal ESIs. ${ }^{24}$ However, the reported rate of neurological sequelae with these injections is extremely low, even when these injections were performed commonly with particulate steroids. The discrepancy of vascular injection frequency to reported clinical sequelae suggests that blood factors may prevent steroid particle aggregation.

We assessed ex vivo the behavior and microscopic appearance of betamethasone and methyl-prednisolone when prepared in water with $1 \%$ lidocaine vs with $0.5 \%$ bupivacaine in a previous study. ${ }^{11}$ The study showed that while there was a significant decrease in the percentage of large steroid aggregates of methylprednisolone in $1 \%$ lidocaine solution, there was an increase in the size of the steroid particle aggregate of methylprednisolone in the $0.5 \%$ bupivacaine serum solution (Table 1 ). Human serum decreased betamethasone aggregates appreciably. Thus, we demonstrated that serum does impact steroid particle aggregation.

Triamcinolone was not evaluated in our previous manuscript, but we introduce its serum aggregation characteristics here. Triamcinolone ESIs have the highest adverse event rate of any of the particulate steroids. CNS ischemic infarctions, blindness, and death have all been reported. Its aggregation characteristics have not been described in physiologic solution. ${ }^{2,16,25-28}$ We postulated, therefore, that triamcinolone would aggregate similar, or more so, in human serum compared to non-physiologic solutions evaluated by other investigators. ${ }^{7,8}$ In this study, we examined triamcinolone in serum, ex vivo, in lidocaine $1 \%$, bupivacaine $0.5 \%$, and water solution. There was a significant increase in the steroid 


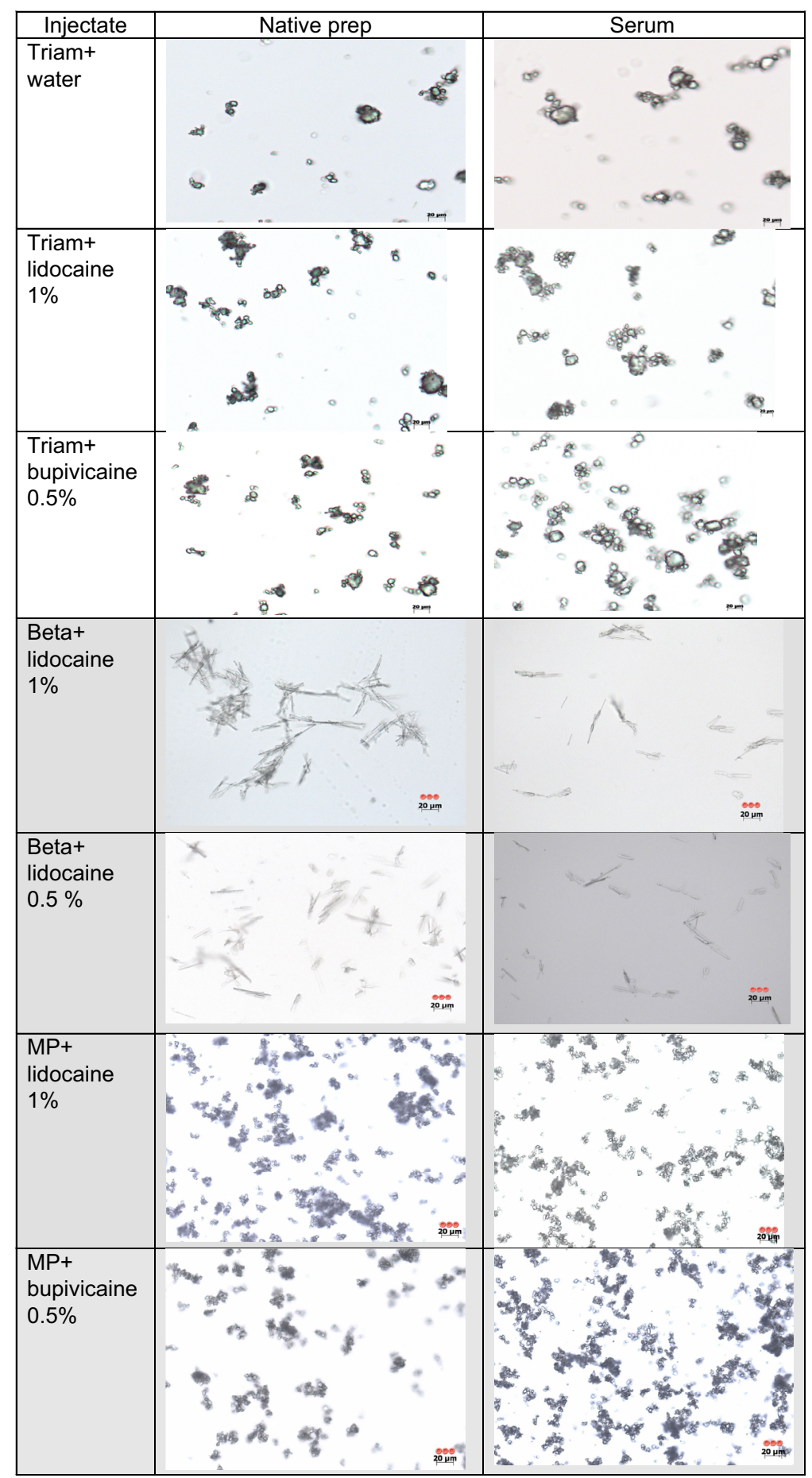

Figure I 400x images of triamcinolone (Triam), betamethasone (Beta), and methyl-prednisilone (MP) in three preparations (prep), side-by-side comparison of native vs human serum.

Note: Shaded rows are from our previous study and are displayed for comparative purposes.

particle aggregate size in the serum/triamcinolone/lidocaine $1 \%$ solution. This aggregation may be clinically significant, as the average size of the steroid particle aggregate $70 \mu \mathrm{m}$; the average diameter of an arteriole measures $37 \mu \mathrm{m}$. This strongly suggests that this steroid-anesthetic mixture may have an increased risk of particle embolism, potentially having neurologic sequelae. Moreover, the comparative size of triamcinolone particulates of betamethasone and methylprednisolone in the same anesthetic and serum solutions identifies triamcinolone as a greater risk of occlusion because 


\begin{tabular}{|c|c|c|c|c|c|}
\hline Solutions & Singlets & Doublets & Triplets & Quadruplets & $>4$ \\
\hline $\mathrm{L} 1 \%$ w serum Triam 400x & 7 & 6.6 & 8.6 & 8 & 38 \\
\hline $\mathrm{B} 0.5 \%$ w serum Triam 400x & 19 & 8 & 9 & 10.3333333 & 46 \\
\hline $\mathrm{H}_{2} \mathrm{O}$ w serum Triam $400 \times$ & 16.8 & 7.8 & 10.4 & 7.8 & 31 \\
\hline $\mathrm{H}_{2} \mathrm{O}$ only Triam $400 \times$ & 5 & 1.9 & 2.1 & 3.5222222 & 8 \\
\hline
\end{tabular}

Aggregation of triamcinolone crystals in different mediums

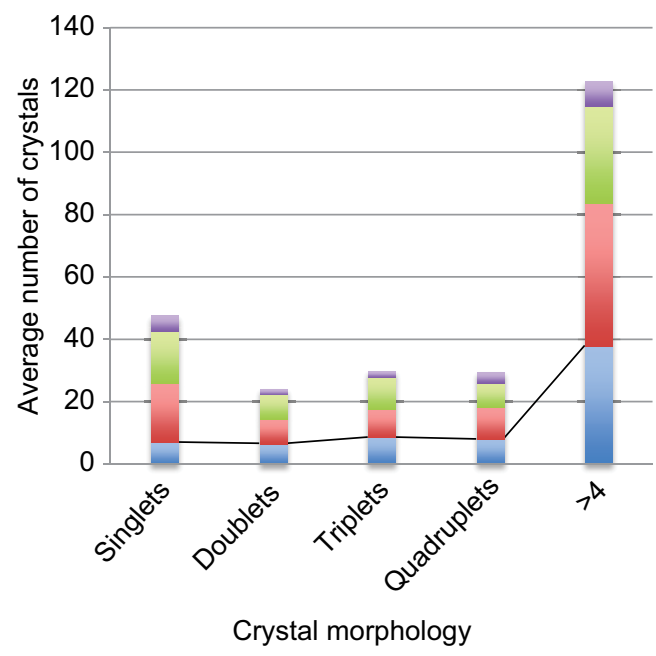

$\mathrm{H}_{2} \mathrm{O}$ only Triam $400 \times$

$\mathrm{H}_{2} \mathrm{O}$ w serum Triam 400x

B0.5\% w serum Triam 400x

- L1\% w Serum Triam 400x

Figure 2 Triam aggregate in anesthetic solution with serum and without serum.

Abbreviations: B, betamethasone; L, lidocaine; Triam, triamcinolone.

it showed the largest relative aggregates seen in physiologic solution. ${ }^{11}$ This is in accord with clinical reports that link triamcinolone with the greatest incidence of devastating embolic phenomena.

The authors suggest that if triamcinolone is used for ESIs, and if local anesthetic is preferred, that the local anesthetic and triamcinolone be injected separately. In addition, for all cases of triamcinolone, we suggest using digital subtraction whenever available; if not, injecting under live fluoroscopy to define lack of vascular detail is also suggested. We believe that the use of extension tubing should be used at the discretion of the practitioner because tubing has an inherent risk of altering final needle tip position (perhaps into a vessel) because the natural tension in packed, coiled tubing can displace preferred needle location. The use of a blunt needle is recommended whenever available. It is not unreasonable to perform a physiologic test injection with local anesthetic and epinephrine to assess intravascular injection prior to steroid injection. Thoughtful and careful practice should be mandated prior to injecting any particulate steroid.

A limitation of this study was its ex vivo preparation in predicting the in vivo behavior of the steroid-anesthetic solution in serum rather than whole blood. While examining the steroid behavior in blood is scientifically optimal, it hindered clear viewing of the steroid particles upon trial examinations preceding our serum analysis. We do not know why serum had a clumping effect on some steroids but not others; further research is required to understand this better. If the agents that cause steroid aggregation can be identified perhaps a solution can be created to retard, or reverse, this process.

Another limitation was variability of blood $\mathrm{pH}$ or osmolality, which may have impacted the steroid particle behavior and was not controlled in this study. Blood was drawn via venipuncture but clinically apparent neurologic sequelae occur as a cause of arterial disruption. We assumed that venous blood would create the same effect to steroid aggregation as arterial blood. Future investigation may warrant the evaluation of arterial blood and steroid aggregation. Lastly, our study was designed to assess triamcinolone aggregation in commonly used mixtures by pain physicians to complement previously studied methylprednisolone and betamethasone, focusing on the particle size as the component of morbidity with the assumption that embolism is the mechanism of devastating neuro-sequelae. ${ }^{11}$ However, we recognize that some investigators doubt embolism as the exact mechanism of injury to the brain or spinal cord following spinal injection. ${ }^{6}$ 
This study was based on the assumption that embolization of particulate steroids used in ESIs was the mechanism by which the observed neurological sequelae occurred. However, we recognize that several other theories exist to explain this phenomenon. ${ }^{6}$

\section{Conclusion}

Comparing triamcinolone behavior in different serum-based preparations showed that serum increases the size of the triamcinolone aggregate. We demonstrated a significant increase in the triamcinolone aggregate size when mixed with lidocaine $1 \%$ compared to bupivacaine $0.5 \%$. When comparing the large crystal aggregates of triamcinolone to betamethasone and methylprednisolone, triamcinolone aggregate size ranged from 70 to $160 \mu \mathrm{m}$, compared to 40 to $100 \mu \mathrm{m}$ for betamethasone and methylprednisolone in our previous study. Here, we demonstrated that triamcinolone may become more aggregate if injected into a blood vessel compared to its already large particulates in non-physiologic solution. This should make the pain physician wary about injection in artery-rich spinal locations.

In clinical setting, where a particulate steroid and local anesthetic are preferred for spinal injections, we suggest that betamethasone and $0.5 \%$ bupivacaine may pose the least risk in artery clotting based on our ex vivo model. In the event the provider prefers using triamcinolone, which we believe forms the largest aggregate size compared to other steroids, triamcinolone mixed with bupivacaine $0.5 \%$ produces smaller size aggregates compared to lidocaine $1 \%$. Nevertheless, high-risk injectable anatomical locations (cervical $>$ thoracic $>$ lumbar transforminals) should be considered by the provider in the clinical setting regardless of what steroid is being used.

\section{Disclosure}

The authors report no conflicts of interest in this work.

\section{References}

1. Mcgrath JM, Schaefer MP, Malkamaki DM. Incidence and characteristics of complications from epidural steroid injections. Pain Med. 2011;12(5):726-731.

2. Tripathi M, Nath SS, Gupta RK. Paraplegia after intracord injection during attempted epidural steroid injection in an awake-patient. Anesth Analg. 2005;101(4):1209-1211.

3. Derby R, Lee SH, Kim BJ, Chen Y, Seo KS. Complications following cervical epidural steroid injections by expert interventionalists in 2003 . Pain Physician. 2004;7(4):445-449.

4. Huntoon MA. Anatomy of the cervical intervertebral foramina: vulnerable arteries and ischemic neurologic injuries after transforaminal epidural injections. Pain. 2005;117(1):104-111.

5. Suh DC, Kim SJ, Jung SM, et al. MRI in presumed cervical anterior spinal artery territory infarcts. Neuroradiology. 1996;38(1):56-58.

6. Gharibo C, Koo C, Chung J, Moroz A. Epidural steroid injections: an update on mechanisms of injury and safety. Techn Reg Anesth Pain Manag. 2009;13(4):266-271.
7. Derby R, Lee SH, Date ES, Lee JH, Lee CH. Size and aggregation of corticosteroids used for epidural injections. Pain Med. 2008;9(2):227-234.

8. Benzon HT, Chew TL, McCarthy RJ, Benzon HA, Walega DR. Comparison of the particle sizes of different steroids and the effect of dilution: a review of the relative neurotoxicities of the steroids. Anesthesiology. 2007; 106:331-338.

9. Gazelka HM, Burgher AH, Huntoon MA, Mantilla CB, Hoelzer BC. Determination of the particulate size and aggregation of clonidine and corticosteroids for epidural steroid injection. Pain Physician . 2012;15:87-93.

10. Ballantyne GH. Telerobotic gastrointestinal surgery: phase 2-safety and efficacy. Surg Endosc. 2007;21(7):1054-1062.

11. Wahezi SE, Lederman A, Algra J, Kim SY, Sellars R. Human serum modifies aggregation properties of commonly used epidural steroids. Pain Physician. 2015;18:E1131-1138.

12. Racoosin JA, Seymour SM, Cascio L, Gill R. Serious neurologic events after epidural glucocorticoid injection - the FDA's risk assessment. $N$ Engl J Med. 2015;373(24):2299-2301.

13. Scanlon GC, Moeller-Bertram T, Romanowsky SM, et al. Cervical transforaminal epidural steroid injections: more dangerous than we think? Spine (Phila Pa 1976). 2007;32(11):1249-1256.

14. Engel A, King W, MacVicar J, et al; Standards Division of the International Spine Intervention Society. The effectiveness and risks of fluoroscopically guided cervical transforaminal injections of steroids: a systematic review with comprehensive analysis of the published data. Pain Med. 2014;15(3):386-402.

15. Manchikanti L, Pampati V, Boswell MV, Smith HS, Hirsch JA. Analysis of the growth of epidural injections and costs in the Medicare population: a comparative evaluation of 1997, 2002, and 2006 data. Pain Physician. 2010;13:199-212.

16. Tiso R, Cutler T, Catania JA, Whalen K. Adverse central nervous system sequelae after selective transforaminal block: the role of corticosteroids. Spine J. 2004;4(4):468-474.

17. Ludwig MA, Burns SP. Spinal cord infarction following cervical transforaminal epidural injection: a case report. Spine (Phila Pa 1976). 2005;30(10): E266-E268.

18. Baker R, Dreyfuss P, Mercer S, Bogduk N. Cervical transforaminal injection of corticosteroids into a radicular artery: a possible mechanism for spinal cord injury. Pain. 2003;103(1-2):211-215.

19. Rathmell JP, Aprill C, Bogduk N. Cervical transforaminal injection of steroids. Anesthesiology. 2004;100(6):1595-1600.

20. Karasek M, Bogduk N. Temporary neurologic deficit after cervical transforaminal injection of local anesthetic. Pain Med. 2004;5(2):202-205.

21. Rathmell JP, Aprill C, Bogduk N. Cervical transforaminal injection of steroids. Anesthesiology. 2004;100:1595-1600.

22. Benzon HT, Chew TL, McCarthy RJ, Benzon HA, Walega DR. Comparison of the particle sizes of different steroids and the effect of dilution: a review of the relative neurotoxicities of the steroids. Anesthesiology. 2007;106(2):331-338.

23. McLean JP, Sigler JD, Plastaras CT, Garvan CW, Rittenberg JD. The rate of detection of intravascular injection in cervical transforaminal epidural steroid injections with and without digital subtraction angiography. $P M$ R. 2009;1(7):636-642.

24. Nahm FS, Lee CJ, Lee SH, et al. Original article: risk of intravascular injection in transforaminal epidural injections. Anaesthesia. 2010;65(9): 917-921.

25. Brouwers PJ, Kottink EJ, Simon MA, Prevo RL. A cervical anterior spinal artery syndrome after diagnostic blockade of the right C6-nerve root. Pain. 2001;91(3):397-399.

26. Somayaji HS, Saifuddin A, Casey ATH, Briggs TWR. Spinal cord infarction following therapeutic computed tomography-guided left L2 nerve root injection. Spine (Phila Pa 1976). 2005;30(4): E106-E108.

27. Suresh S, Berman J, Connell DA. Cerebellar and brainstem infarction as a complication of CT-guided transforaminal cervical nerve root block. Skeletal Radiol. 2007;36(5):449-452.

28. Lyders EM, Morris PP. A case of spinal cord infarction following lumbar transforaminal epidural steroid injection: MR imaging and angiographic findings. AJNR Am J Neuroradiol. 2009;30(9):1691-1693. 
The Journal of Pain Research is an international, peer reviewed, open access, online journal that welcomes laboratory and clinical findings in the fields of pain research and the prevention and management of pain. Original research, reviews, symposium reports, hypothesis formation and commentaries are all considered for publication
The manuscript management system is completely online and includes a very quick and fair peer-review system, which is all easy to use. Visit http://www.dovepress.com/testimonials.php to read real quotes from published authors.

Submit your manuscript here: https://www.dovepress.com/journal-of-pain-research-journal 